\title{
Violencia en el trabajo hacia los profesionales de enfermería en los servicios de emergencias: revisión integrativa
}

\author{
Pia Contreras Jofre, ${ }^{1}$ Ámbar Valenzuela Solís, ${ }^{1}$ Jessica Pinto Soto, ${ }^{1}$ Natalia Mendoza Ponce, ${ }^{1}$ Fanny \\ López-Alegría ${ }^{1}$
}

Forma de citar

Contreras Jofre P, Valenzuela Solís A, Pinto Soto J, Mendoza Ponce N, López-Alegría F. Violencia en el trabajo hacia los profesionales de enfermería en los servicios de emergencias: revisión integrativa. Rev Panam Salud Publica. 2020;44:e173. https://doi.org/10.26633/RPSP.2020.173

RESUMEN

Objetivo. Conocer la frecuencia de la violencia, tanto física como verbal, hacia los profesionales de enfermería, a nivel mundial en los servicios de emergencias y su impacto en la salud y en el contexto laboral.

Métodos. La investigación fue realizada mediante una revisión integrativa, en febrero de 2020, de las bases bibliográfícas MEDLINE, LILACS, CINAHL y SciELO, utilizando los descriptores "Nurses", "Workplace Violence", "Physical Aggression", "Psychological Aggression", "Stress, Psychological", "Psychological Trauma", "Aggression", "Health Impact Assessment", "Health Impact", "Impacts on Health", "Impact Assessment, Health" y "Occupational Health".

Resultados. De los artículos localizados, 18 fueron incluidos en el análisis. Los estudios confirmaron que los profesionales de la salud más afectados por la violencia eran los profesionales de enfermería y el tipo más común fueron las agresiones verbales, seguidas por la violencia física. El área intrahospitalaria más común donde ocurrían dichas agresiones era el servicio de emergencias. El impacto en la salud de los profesionales de enfermería se correlacionó con el estrés, el agotamiento emocional, la ansiedad y el miedo, entre otros síntomas. Con respecto al impacto laboral, se evidenciaron relaciones indirectas significativas vinculadas con la reducción de la productividad. Todos estos aspectos llevaban a los profesionales de enfermería a considerar el abandono de sus trabajos en los servicios de emergencias.

Conclusiones. Los resultados permitieron identificar un perfil de riesgo de violencia contra los profesionales de enfermería en los servicios de emergencias evidenciado por la alta incidencia de violencia física y verbal que repercutió en la disminución de la productividad en su trabajo y la aparición de síntomas relacionados al síndrome de burnout.

Palabras clave Violencia laboral; enfermeras y enfermeros; evaluación del impacto en la salud; salud laboral; servicios médicos de urgencia.

En 1998, la Organización Internacional del Trabajo (OIT) publicó la primera edición del libro "Violence at Work" (1), en este, se definió a la violencia laboral como "toda acción, incidente o comportamiento que se aparta de lo razonable, mediante la cual una persona es agredida, amenazada, humillada o lesionada por otra en el ejercicio de su actividad profesional o como consecuencia directa de la misma" $(2,3)$. En dicha publicación se evidenció la relevancia, a nivel mundial, del problema de

\footnotetext{
Escuela de Enfermería, Facultad de Enfermería, Universidad Andres Bello,

Santiago, Chile. $₫$ Fanny López-Alegría, Fanny.lopez@usach.cl
} 
la violencia en el contexto laboral, con énfasis en el sector de la atención de salud. Esto, porque casi la cuarta parte de los actos de violencia que se cometen en los lugares de trabajo alrededor del mundo, ocurrían en ese sector, siendo ejecutados, generalmente, por pacientes y visitantes $(2,3)$. Por ello, esta problemática fue la base para que la OIT elaborara las Directrices Marco, con el objetivo de ser utilizadas como una orientación para enfrentar la violencia laboral en el sector de la salud $(2,3)$.

Dentro del personal de salud, el correspondiente a enfermería es el que sufre los mayores riesgos, ya que trabajan en situaciones definidas como "riesgos especiales", como los son el trabajar solos (turnos de noche o en domicilio), o en contacto con público, lo que posibilita riesgos de violencia cada vez más altos $(2,3)$.

Estos actos de violencia se demostraron en un amplio estudio realizado en 2013 con trabajadores de la salud de países latinoamericanos de habla hispana, mediante una encuesta electrónica a usuarios del sitio web Intramed, en el cual se obtuvieron 19 967 encuestas para analizar y, de estos usuarios, 13323 (66,7\%) informaron haber recibido algún tipo de agresión en el último año por parte de los pacientes como consecuencia del desarrollo de su tarea asistencial. El estamento enfermería $(71,6 \%)$ fue, junto a los médicos $(71,3 \%)$, los que más habían recibido algún tipo de agresión. En relación con las áreas de ocupación, fueron los profesionales del área de emergencias los que alcanzaron un porcentaje superior al $79 \%$. Estos resultados han ido en aumento con respecto a la encuesta anterior (2006), en la que un $54,6 \%$ de los profesionales refirieron haber sido agredidos (4).

Lo anteriormente expuesto, ha sido confirmado por una revisión sistemática publicada en 2018, acerca de la violencia ejercida sobre los trabajadores del sector de la salud, en la cual se encontraron 2 aspectos, vinculados con un mayor riesgo de sufrir violencia en el trabajo y relacionados con la naturaleza de la labor: por un lado, trabajar en emergencias y, por otro, pertenecer al estamento de enfermería (5). Es así, que a partir de estas circunstancias, se plantea la siguiente pregunta de investigación: ¿cuál es la frecuencia de las agresiones, tanto físicas como verbales, hacia los profesionales de enfermería a nivel mundial en los servicios de emergencias en los últimos 10 años y su impacto en la salud y en el contexto laboral? El objetivo de esta revisión fue conocer la frecuencia de las agresiones hacía los profesionales de enfermería, tanto físicas como verbales, en los servicios de emergencias y su impacto en la salud y en el contexto laboral.

\section{MÉTODOS}

El estudio fue realizado a través de una revisión integrativa de la literatura correspondiente para responder la pregunta de investigación de acuerdo a las etapas recomendadas por la Colaboración Cochrane, que incluyen: formulación de la pregunta de investigación; establecimiento de los criterios de inclusión y exclusión; localización de los artículos; aplicación de criterios de calidad metodológica; recolección de datos; análisis y presentación de los resultados $(6,7)$.

La pregunta de investigación es sustentada por la estrategia PICoR (Pacientes, Intervención, Comparador y Resultado): Paciente -profesional de enfermería agredido-; Intervención agresiones físicas o verbales contra los profesionales de enfermería en los servicios de emergencias-; Comparación - no fue utilizada en esta revisión, ya que el objetivo no era comparar diferentes intervenciones entre sí-; Resultados -conocer la frecuencia y el impacto de las agresiones en la salud y en el trabajo a los profesionales de enfermería.

Para esta estrategia de búsqueda se utilizaron las bases de datos Medical Literature Analysis and Retrieval System online (MEDLINE), Cumulative Index to Nursing and Allied Health Literature (CINAHL), Literatura Latino-Americana e do Caribe em Ciencias da Saúde (LILACS) y Scientific Electronic Library Online (SciELO) consultadas en el mes de febrero de 2020.

En esta búsqueda se utilizaron descriptores seleccionados en inglés tomados de los Medical Subject Headings (MeSH); en español y portugués en los Descriptores en Ciencias de la Salud (DeCS) junto a los operadores booleanos (AND y OR). Los elementos anteriores fueron utilizados para la siguiente estrategia de búsqueda en MEDLINE y luego se adaptaron a otras bases de datos: Search ((nurses) AND ((()((Workplace Violence) OR ((physical aggression) OR psychological aggression))) OR Stress, Psychological) OR Psychological Trauma) OR Aggression)) AND ((() Health impact assessment) OR Health impact) OR Impacts on Health) OR Impact assessment, health OR Occupational Health).

Posterior a esta búsqueda electrónica, se aplicaron los criterios de inclusión y exclusión. Los criterios de inclusión fueron los artículos de investigación publicados en el periodo comprendido entre 2010 y 2020 en español, inglés y portugués. Entre las características particulares del sujeto en estudio se incluyó el ser profesional de enfermería de los servicios de emergencias. Los criterios de exclusión fueron: revisiones narrativas, revisiones sistemáticas, artículos de metodología cualitativa, cartas al editor, artículos de opinión, relatos de experiencia, casos clínicos, libros, presentaciones en congresos, estudios en los que no se apreciaba con claridad el método de medición de la variable en estudio y artículos que no respondían la pregunta de investigación. Entre las características particulares se excluyeron las auxiliares o técnicos o asistentes de enfermería o paramédicos y las agresiones recibidas fuera del ámbito laboral.

Una vez identificados los artículos en las bases de datos, se procedió a la eliminación de los registros duplicados mediante el software EndNoteBasic, Thomson Reuters, USA. A continuación, para refinar más la estrategia de búsqueda, se procedió a ejecutar 2 fases de eliminación de registros: la primera, se hizo mediante la lectura de títulos y resúmenes y la segunda, a través de la lectura a texto completo. A este número de artículos seleccionados a texto completo, se aplicaron los criterios de evaluación de calidad metodológica de los estudios, mediante la escala Strenghening the Reporting of Observational Studies in Epidemiology (STROBE) para los estudios observacionales. Los artículos que obtuvieron una puntuación inferior a $80 \%$ fueron eliminados, obteniéndose en esta última fase los artículos incluidos para una revisión. Todo este proceso de identificación, selección y evaluación de la elegibilidad de los artículos se realizó mediante la utilización del protocolo Preferred Reporting Items for Systematic Reviews and Meta-Analyse (PRISMA) (8).

El análisis de los artículos seleccionados fue realizado por 2 autores de forma independiente y las divergencias fueron resueltas a través de un consenso después de una nueva revisión del o los artículos en discordancia. Este análisis fue hecho mediante una revisión descriptiva y analítica de los resultados encontrados en la lectura de los artículos a texto completo. Los resultados fueron presentados y evaluados, en cuanto a su aplicabilidad o respuesta a la pregunta de investigación, en un cuadro sinóptico que consideró los siguientes aspectos: 
identificación del artículo y el aporte a la pregunta de investigación (porcentaje y tipos de agresiones e impacto en la salud y en el trabajo).

Respecto a los aspectos éticos, fue respetada la integridad de los artículos, es decir, no se realizaron modificaciones de los contenidos encontrados.

\section{RESULTADOS}

Fueron identificados un total de 1341 estudios al aplicar la estrategia de búsqueda en las bases de datos Medline, LILACS, CINAHL y SciELO. La trayectoria de identificación, selección, elegibilidad e inclusión de los estudios seleccionados se puede visualizar en la figura 1.

Los artículos incluidos en el estudio para su análisis fueron 18 (9-26) (cuadro 1). La mayoría de ellos 16 (88,9\%) fueron obtenidos desde la base de datos bibliográfica MEDLINE y el idioma predominante fue el inglés 17 (94,4\%) (9-19, 21-26). Los estudios se realizaron en diversos países como Estados Unidos (9-11), España $(17,20,21)$, Jordania $(16,18)$ y en otros países de África, Asia y Europa, con 1 estudio cada uno (12-15,19,22-26). La mayoría de los artículos fueron publicados entre los años 2015 y 2018 (12 artículos) (13-24). Los tipos de estudio fueron: 15 de tipo transversal cuantitativo descriptivo o correlacional $(9,10,12-14,16-19,21-26), 2$ comparativos $(15,20)$ y 1 estudio prospectivo. (11)

Entre las características particulares de los sujetos de estudio, la población total fue de 2636 profesionales de enfermería y 10 924 personas del equipo de salud (dentro del cual no se definía el número de profesionales de enfermería). En los artículos en los que estaba bien definido el número de profesionales de enfermería, el menor número de ellos fue de 62 y trabajaban en 8 servicios de emergencias en el noreste de Italia (25). El artículo que tuvo el mayor número de profesionales de enfermería fue de 266, estos trabajaban en los servicios de emergencias de 4 de los más grandes hospitales de Karachi, Pakistán (12).

La mayoría de la población de estudio fueron mujeres y el artículo que refiere la menor proporción de ellas fue de $46,3 \%$ (25), mientras que el estudio que tuvo mayor proporción fue de $80,5 \%$ enfermeras, estas últimas fueron miembros de la Asociación de Enfermeras Servicio de Emergencias de Estados Unidos (9). La población de estudio era joven, con una edad promedio que iba desde 27,1 a 44,5 años (10-13,16-19,21,25). El tiempo que los profesionales de enfermería trabajaron en los servicios de emergencias varió desde 6,6 a 30 años $(11,13,15,24,25)$.

Esta revisión confirmó que los profesionales de la salud más afectados son, precisamente, los profesionales de enfermería $(9-11,14,20,21,25)$ y que las agresiones contra ellos tienen una frecuencia que va desde un $13,4 \%$ a un $95,3 \%$ de la población de estudio $(16,23)$. Dentro del equipo de salud, los profesionales de enfermería reportaron el número de eventos de violencia más altos (0-61 $\pm 0,05$ evento por mes por persona) (11). Con respecto a estas agresiones se constató que la violencia de tipo verbal es muy superior a la física, siendo que en algunos casos la verbal fue superior cinco veces a la física (10-12,14-17,20,22-25). Los protagonistas principales de estos hechos son los pacientes, que, en algunos casos, llegaron a participar en un $82 \%$ de los eventos de violencia $(10,22,24,25)$ o también sus familiares, en porcentajes que llegaron hasta el 85,4\% de los casos $(12-14,22,24,25)$. Llegando en algunos casos, estas actitudes violentas, a una intervención policial, como lo describe el estudio en el servicio
FIGURA 1. Flujograma de identificación, selección, elegibilidad e inclusión de los estudios
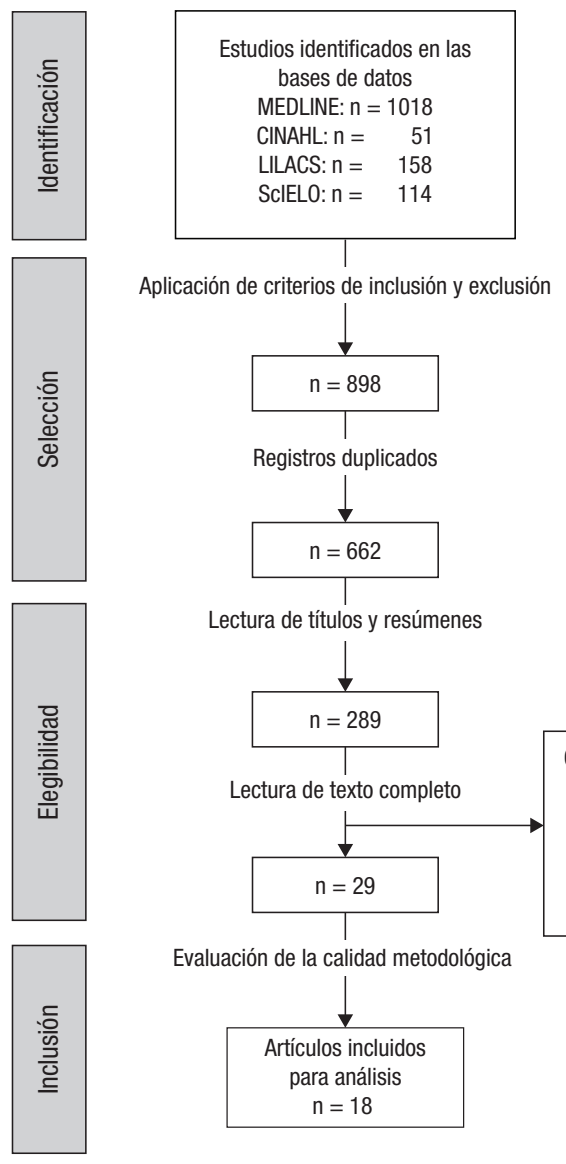

Fuente: elaboración propia a partir de los resultados presentados en este estudio.

de emergencias del Hospital de Bahréin (32\% de los casos de violencia) (22). Los tipos de violencia no física se caracterizaban por ser insultos, amenazas y acoso sexual (14); en tanto, las agresiones físicas se caracterizaban como patadas, el arrojar instrumentos y el uso de armas, siendo los empujones los más comunes $(75 \%)(14,23)$.

En el ámbito hospitalario, las áreas más comunes en que sucedían los hechos de violencia fueron los servicios de emergencias (9-26). Ogenler y cols., demostraron una relación significativa entre la violencia física y la unidad de trabajo, confirmando que la unidad de emergencias era el lugar más frecuente donde se manifestaba este tipo de violencia (23). Dos estudios de tipo comparativo fueron realizados para evaluar la prevalencia de la violencia en diversos servicios hospitalarios, uno en Egipto en 2013 y el otro en España con datos del 2009 al 2014. En ambos se reafirmó que el servicio de emergencias es el que tiene más evento de violencia contra los profesionales de enfermería $(15,20)$.

El reporte o denuncia de esta violencia, en la mayoría de las veces, fue escasamente realizado $(10,14,18,23,24)$ u otras veces fue reportado en un alto porcentaje $(95,3 \%)$ de los casos revisados. (16) Las razones informadas para justificar el bajo nivel de denuncia fueron atribuidas a los procedimientos legales no cumplidos, informes no considerados, el hecho de tener que manejar el incidente personalmente y el miedo a las 
$9 \quad$ El 94\% de los profesionales de enfermería sufrió al menos 1 síntoma de stress después del evento violencia.

El 37\% de los profesionales de enfermería tenían un puntaje de la Escala HPS de productividad negativo (disminución de la productividad después del evento). El 17\% tenía puntajes altos de la escala IES_R que indicaban un probable estrés postraumático.

Todas las escalas, excepto Seguridad y Cuidados, tenían puntaje negativo, indicando una disminución del rendimiento laboral.

Un 40\% experimentaron sobre 10 episodios de amenazas verbales y un 68\%, por lo menos, una amenaza física por parte de los pacientes.

El promedio de la Escala de seguridad fue 16,17 (rango 3 -30). El 54\% se sentía inseguro trabajando en el servicio de emergencia. Aunque no fue estadísticamente significativo los profesionales de enfermería tenían más altos puntajes (sentirse menos segura) en la Escala de seguridad ( $X=17,69)$ que los asistentes de enfermería $(X=15,42)$ y que los médicos $(X=13,29)$.

11 La tasa promedio de violencia por persona fue de 4,15. Se informaron diferencias significativas en las violencia entre enfermeros/as y médicos $(P=.0017)$ y asistentes $(\mathrm{P}<.05)$. Los profesionales de enfermería tenían más probabilidades de experimentar estrés agudo que los médicos $(\mathrm{P}<.001)$. El estrés agudo redujo la productividad $(P<.001)$. Los profesionales de enfermería reportaron el número de eventos de violencia más altos $(0-61 \pm 0,05$ evento por mes por persona).

12 El 16,5\% (médicos y profesionales de enfermería) dijeron que habían sido atacados físicamente y el 29,6\% de estos, informó que el incidente involucró un arma. La violencia se asoció con efectos sobre la salud mental en forma de recuerdos molestos, super-alerta y sentimientos de evitación e inutilidad.

13 El 76,2\% (profesionales de enfermería y médicos) estuvieron expuestos al abuso verbal. La falta de información (72,2\%) y la creencia de que la violencia en el lugar de trabajo es parte del trabajo $(74,1 \%)$ son altas.

14 El 76,1\% del equipo de salud había experimentado un tipo de violencia. El personal más joven (OR =2,29 IC 95\% 1309-4036), los profesionales de enfermería y médicos (OR = 1,65 IC 95\% 0,979-2,797) en comparación con el personal administrativo y con menos experiencia en el servicio de urgencias (OR $=2,39$ IC 95\% 1141-5006) tienen un riesgo significativamente mayor de exposición a la violencia en los servicios de urgencia $(\mathrm{P}<0,05)$.

15 El $85,9 \%$ de los profesionales de enfermería de los servicios de emergencias han tenido uno o más episodios de violencia en el trabajo comparado con 79,6\% de los servicios de medicina interna.

16 El $77 \%$ de los profesionales de enfermería se sentían inseguras en el servicio de emergencias y solo el $41,4 \%$ informaron haber aprendido a manejar situaciones de violencia en el lugar de trabajo. El 51,7\% de los profesionales de enfermería s informaron de estos incidentes a través de declaraciones formales escritas y de estos el $69,5 \%$ de los profesionales de enfermería informaron satisfacción con los procedimientos de denuncia. Además, el $69,8 \%$ de los participantes del estudio informaron haber tomado licencias por enfermedad como resultado de la investigación.

17 Los niveles del Síndrome de Burnout eran $0 \%$ en los profesionales que no habían sufrido violencia y este subía a 6,7\% en los que habían sufrido violencia, siendo estadísticamente significativo.

18 La sala de tratamiento era el lugar más común donde ocurría la violencia. Solo el 16,6\% de los profesionales de enfermería que experimentaron violencia lo denunciaron. Estar acostumbrado/a a la violencia en el lugar de trabajo es la razón más declarada para no denunciar los hechos de violencia a las autoridades del hospital.

19 Un 88\% de los profesionales de enfermería habían sufrido violencia física o verbal por los pacientes.

La resiliencia y el apoyo de los pares fueron, significativamente, mayores en el grupo sin tendencia a la depresión. Los componentes de la resiliencia como la fuerza personal, la competencia social, el estilo de estructura y las creencias religiosas fueron factores significativos que representaron el $46 \%$ de la variación en la tendencia depresiva.

20 El estudio se dividió en trabajadores de atención primaria (AP) y atención especializada (AE) que incluye los servicios de urgencias. Del equipo de salud, los profesionales de enfermería son los más agredidas en AE $(47,4 \%)$ y los médicos en AP $(53.1 \%)(p<0,001)$.

Del daño psicológico, el $42,9 \%$ fue grave en AP y 14,3\% en AE. De las lesiones físicas el 5,6\% fue de tipo grave en AP y el 1,6\% en AE.

Se encontró una correlación negativa entre la dimensión de violencia no física y la tenencia en la profesión $(r=-.09, p<.05)$, es decir, una mayor experiencia se relacionó con un menor riesgo de sufrir violencia verbal. Además, se encontraron diferencias entre la violencia no física y el nivel profesional, $t=2,86$, $d f=503, p<.01, d=0,333$, con niveles más altos de violencia recibidos por las enfermeras que las auxiliares. La dimensión de violencia no física del HABS-U se correlacionó positivamente con el agotamiento emocional del MBI-GS $(r=.27, p<.01)$ y con el GHQ-28.

Las víctimas de violencia reportaron sentirse emocionalmente agotadas (Síndrome de Burnout). El $26 \%$ de la muestra había considerado abandonar el trabajo en el servicio de emergencias, también se registró un bajo rendimiento en lo que respecta a logros personales.

23 El $44,0 \%$ de las víctimas de violencia informaron el acto de violencia al jefe de su unidad, $18,0 \%$ no hicieron nada y el 6,0\% llevaron el asunto a la corte. Hubo una asociación significativa entre la violencia física y los niveles de ansiedad.

$2442,6 \%$ reportó procedimientos de violencia. Un 92,1\% de profesionales de enfermería que presenciaron incidentes violentos no los reportaron. Expresando que "la violencia no física es parte del trabajo".

25 El tipo de violencia más frecuente es la emocional perpetrada por los pacientes Los profesionales de enfermería Los/as enfermeros/as reciben mayor violencia que los médicos, por la cercanía con los pacientes.

26 La prevalencia de violencia fue de 47,9\% entre médicos y los profesionales de enfermería . Destacaban síntomas de depresión y ansiedad en médicos (58,8\%) y enfermeros/as (39,7\%).

Fuente: elaboración propia a partir de los resultados presentados en este estudio.

consecuencias. $(13,14,16)$ Un aspecto preocupante, fue el hecho verificado en 4 artículos, en el cual los profesionales de enfermería refirieron que la violencia era aceptada como parte del trabajo, $(13,18,22,24)$ siendo esta, en algunos casos, la razón más declarada para no denunciar estos hechos de violencia a la administración del hospital o a las autoridades. (18) Además, 3 artículos refieren que más de la mitad de los profesionales de enfermería no tenían entrenamiento para la prevención de estos actos en su lugar de trabajo $(10,11,23)$.
Con respecto a las causas de la violencia mencionada por el equipo de salud, se establecen: el tiempo de espera, la intoxicación por alcohol, el abuso de sustancias, ciertas enfermedades mentales, las expectativas insatisfechas de los pacientes y la falta de personal de seguridad $(13,14,18,22)$.

Los instrumentos de evaluación utilizados para medir la violencia en el lugar de trabajo fueron: Cuestionario de salud general (GHQ-28) (17,21), Maslach Burnout Inventory (MBI) (mide la presencia del cansancio emocional, la 
despersonalización y la falta de realización personal), $(17,21)$ Impact of Events Scale-Revised ${ }^{\circ}$-IES-R (evalúa la presencia y magnitud de síntomas de estrés postraumático), (9) Healthcare Productivity Survey -HPS (mide la percepción de cambio en la productividad laboral después de una exposición a un evento estresante), (9) Workplace Violence in the Health Sector Case Study Research Instruments Survey Questionnaire (Encuesta del Programa Conjunto sobre Violencia en el Lugar de Trabajo en el Sector de la Salud de la Oficina Internacional del Trabajo, el Consejo Internacional de Enfermeras, la Organización Mundial de la Salud y los Servicios Públicos Internacionales) (12,14,15, 24). Sin embargo, en 4 artículos se trabajó con instrumentos creados por el propio autor $(10,11,22,23)$.

Con relación al impacto que tuvo la violencia en la salud de los profesionales de enfermería, se observó que la salud mental fue la más afectada, con argumentos referidos como "recuerdos molestos", "sentimientos de evitación", "ansiedad", "estrés", "agotamiento emocional", y que tuvo como consecuencia final el padecer el síndrome de burnout $(11,12,17,20-23,26)$. El impacto que tuvo la violencia no física fue medida por la Escala de Comportamiento Agresivo de Trabajadores Sanitarios (HABS-U), se correlacionó positivamente con el agotamiento emocional medido por Maslach Burnout Inventory - General Survey (MBI-GS) ( $\mathrm{r}=.27, \mathrm{p}<.01)$ y el General Health Questionnaire (GHQ-28) (17). Este estudio fue realizado con enfermeras/os de 70 servicios de emergencias de España y mostró que los niveles del síndrome de Burnout eran $0 \%$ en los profesionales que no habían sufrido violencia y que subía a $6,7 \%$ en los que sí habían sufrido violencia verbal y física, lo cual era estadísticamente significativo (17).

En relación al impacto de la violencia en el contexto laboral, la inseguridad en el lugar de trabajo fue lo más prevalente, llegando en algunos estudios al 77\% de las enfermeras a referir este evento $(9-11,16)$. La escala Healthcare Productivity Survey (HPS) fue utilizada para medir la productividad después de los episodios de violencia de un grupo de 230 profesionales de enfermería miembros de la Asociación de Enfermeras Servicio de Emergencias de Estados Unidos, obteniendo un puntaje promedio total de productividad negativo $(-0,05)$, es decir, se produjo una disminución de la productividad (9).

\section{DISCUSIÓN}

Ningún profesional de enfermería debiera sufrir agresiones en el ejercicio de sus funciones laborales, ya que la violencia es inaceptable. Esta es la respuesta que debería darse a la pregunta de investigación planteada en este estudio, pero la realidad es diferente. La violencia contra el personal de salud ha aumentado casi un 110 por ciento en los últimos 10 años, según el Instituto Nacional de Seguridad y Salud Ocupacional (27). Esto, ha hecho que se considere esta situación como un problema de salud pública mundial, $(2,3,29)$, afirmación que se ve respaldada por los datos recogidos en esta revisión integrativa que incluye a 12 países de 4 continentes (9-26).

Los artículos analizados en esta revisión confirman el perfil de riesgo de violencia contra los profesionales de enfermería, identificando las causas involucradas en este tipo de acciones, el estamento profesional más aquejado, los servicios hospitalarios más afectados, las circunstancias desencadenantes de la violencia y los tipos de repercusiones en la salud y en el trabajo. Todos estos aspectos son similares en las diversas regiones geográficas (9-26) y este perfil es equivalente a 4 revisiones sistemáticas publicadas entre los años 2011 y 2018 por autores de Canadá, Chile, Estados Unidos, e Irán (5,28-30). Esta identificación y confirmación de dicho perfil contribuye a una mayor comprensión del fenómeno en estudio.

Frente a esta problemática ha existido una preocupación por parte de instituciones como la Organización Mundial de la Salud (OMS), la Organización Internacional del Trabajo (OIT), el Consejo Internacional de Enfermería y los Servicios Públicos Internacionales, entre las principales, que desde la década del 2000 han intentado disminuir o velar por reducir la frecuencia y gravedad de estos eventos mediante la regulación e instauración de directrices que guíen el manejo de este tipo de violencia $(2,3,31)$. Sin embargo, y al parecer, las estrategias definidas en las Directrices Marco para gestionar y hacer frente a la violencia no han ayudado a las víctimas frente a estas agresiones (3), ya que la alta prevalencia de la violencia sigue siendo similar una década después $(87,4 \%)$, lo que queda demostrado en el trabajo de Al-Maskari SA y cols. del 2020 (32). Para contribuir con estas instituciones, desde el punto de vista de la investigación científica y que aparece como limitaciones inherentes de esta revisión, es que las investigaciones empleen instrumentos uniformes, validados y confiables para comparar el evento en estudio, pues el propósito de una revisión sistemática es integrar y evaluar evidencias $(9,12,14,15,17,21,24)$. Esta contribución científica, unido al trabajo de estas instituciones y los servicios de salud involucrados, pueden aportar a una optimización de la gestión del problema de la violencia laboral contra estos profesionales.

En lo que respecta a las limitaciones del estudio, se demostró que al medir la extensión de la violencia en el ambiente laboral se advierte una subnotificación, ya que se evidencia que los profesionales en estudio no tienen un concepto claro y consciente de la definición de violencia, lo que dificulta el diagnóstico real. El creer que estos incidentes "formar parte del trabajo" y que no constituyen violencia $(24,34)$ ha permitido que las agresiones sean integradas y que se perpetúen como actos normales dentro del contexto laboral $(18,24,33,34)$. Esta cultura de aceptación de la violencia como parte del trabajo ha llegado a niveles altos $(95,3 \%)$ y tiene entre sus consecuencias el bajo nivel de denuncia de estos hechos $(40 \%)(13,16,23,33,34)$. Como fortaleza se señala que los datos vienen de estudios poblacionales con un gran número de participantes en estudio, pertenecientes a diversas regiones geográficas y de instituciones que están permanentemente encargadas de recopilar estos datos $(9,20)$.

En conclusión, los estudios revisados identificaron un perfil de riesgo de violencia principalmente dirigida contra los profesionales de enfermería de los servicios de emergencias, que se evidenció por la alta incidencia de agresiones tanto física como verbal, lo que repercutió en una disminución de la productividad laboral y el surgimiento de síntomas relacionados con del síndrome de Burnout.

Como recomendaciones se sugiere que para tener una imagen real del fenómeno en estudio y evitar la subnotificación se debe modificar la actitud del personal hacía el concepto de violencia y su denuncia, que sería el primer paso para disminuir la incidencia de este problema. Lo anterior, podría ser optimizado poniendo énfasis en las leyes de protección del personal y en la capacitación continua para los funcionarios; además, de una educación comunitaria dirigida a los pacientes, que incluya información sobre los derechos y deberes de ellos. 
Contribución de los autores. Todos los autores PCJ, AVS, JPS, NMP, FLA concibieron el estudio original, recolectaron y analizaron los datos, interpretaron los resultados y escribieron y revisaron el manuscrito. Todos los autores revisaron y aprobaron la versión final.
Conflicto de intereses. Ninguno declarado por los autores.

Declaración. Las opiniones expresadas en este manuscrito son responsabilidad del autor y no reflejan necesariamente los criterios ni la política de la $R P S P / P A J P H$ y / o de la OPS

\section{REFERENCIAS}

1. Chappell D, Di Martino V. Violence at Work. Geneva, International Labour Office, 1998. 165 pp.

2. Organización Internacional del Trabajo. Consejo Internacional de Enfermeras. Organización Mundial de la Salud. Internacional de Servicios Públicos. Directrices marco para afrontar la violencia en el sector de la salud. Ginebra. Organización Internacional del Trabajo, 2002. 38 Pp.

3. Organización Internacional del Trabajo: Programa de actividades sectoriales. Repertorio de recomendaciones prácticas sobre la violencia en el lugar de trabajo en el sector de los servicios y medidas para combatirla. Reunión de expertos para elaborar un repertorio de recomendaciones prácticas sobre la violencia y el estrés en el trabajo en el sector de los servicios: una amenaza para la productividad y el trabajo decente. 8-15 de octubre de 2003. 28 pp.

4. Travetto C, Daciuk N, Fernández S, Ortiz P, Mastandueno R, Prats $\mathrm{M}$ et al. Agresiones hacia profesionales en el ámbito de la salud. Rev Panam Salud Pública. 2015;38(4):307-15.

5. Palma A, Ansoleaga E, Ahumada M. Violencia laboral en trabajadores del sector salud: revisión sistemática. Rev Med Chil. 2018 Feb;146(2):213-222. doi: 10.4067/s0034-98872018000200213.

6. Barbosa FT, Lira AB, Oliveira Neto OB, Santos LL, Santos IO, Barbosa LT, et al. Tutorial for performing systematic review and meta-analysis with interventional anesthesia studies. Rev Bras Anestesiol. 2019 May - Jun;69(3):299-306. doi: 10.1016/j.bjan.2018.11.007.

7. Higgins JPT, Green S (editors). Cochrane Handbook for Systematic Reviews of Interventions Version 5.1.0 [updated March 2011]. The Cochrane Collaboration, 2011. Disponible: www.cochranehandbook.org. Acceso el día 10 febrero 2020.

8. Moher D, Shamseer L, Clarke M, Ghersi D, Liberati A, Petticrew M, et PRISMA-P Group. Preferred reporting items for systematic review and meta-analysis protocols (PRISMA-P) 2015 statement. Syst Rev. 2015 Jan 1;4:1. doi: 10.1186/2046-4053-4-1.

9. Gates DM, Gillespie GL, Succop P. Violence against nurses and its impact on stress and productivity. Nurs Econ. 2011 Mar-Apr; 29(2):59-66.

10. Gates D, Gillespie G, Kowalenko T, Succop P, Sanker M, Farra S. Occupational and demographic factors associated with violence in the emergency department. Adv Emerg Nurs J. 2011 Oct-Dec;33(4):303-13. doi: 10.1097/TME.0b013e3182330530.

11. Kowalenko T, Gates D, Gillespie GL, Succop P, Mentzel TK. Prospective study of violence against ED workers. Am J Emerg Med. 2013 Jan;31(1):197-205. doi: 10.1016/j.ajem.2012.07.010

12. Zafar W, Siddiqui E, Ejaz K, Shehzad MU, Khan UR, Jamali S et al. Health care personnel and workplace violence in the emergency departments of a volatile metropolis: results from Karachi, Pakistan. J Emerg Med. 2013 Nov;45(5):761-72. doi: 10.1016/j. jemermed.2013.04.049

13. Vezyridis P, Samoutis A, Mavrikiou P. Workplace violence against clinicians in Cypriot emergency departments: a national questionnaire survey. J Clin Nurs. 2015 May;24(9-10):1210-22. doi: 10.1111/ jocn.12660.

14. Hamdan M, Abu Hamra A. Workplace violence towards workers in the emergency departments of Palestinian hospitals: a cross-sectional study. Hum Resour Health. 2015 May 7;13:28. doi: 10.1186/ s12960-015-0018-2.

15. Abou-ElWafa HS, El-Gilany AH, Abd-El-Raouf SE, Abd-Elmouty SM, El-Sayed Rel-S. Workplace violence against emergency versus non-emergency nurses in Mansoura university hospitals, Egypt. J Interpers Violence. 2015 Mar;30(5):857-72. doi: $10.1177 / 0886260514536278$
16. Darawad MW, Al-Hussami M, Saleh AM, Mustafa WM, Odeh H. Violence against nurses in emergency departments in Jordan: nurses' perspective. Workplace Health Saf. 2015 Jan;63(1):9-17. doi: $10.1177 / 2165079914565348$.

17. Bernaldo-De-Quirós M, Piccini AT, Gómez MM, Cerdeira JC. Psychological consequences of aggression in pre-hospital emergency care: cross sectional survey. Int J Nurs Stud. 2015 Jan;52(1):260-70. doi: 10.1016/j.ijnurstu.2014.05.011.

18. ALBashtawy M, Aljezawi M. Emergency nurses' perspective of workplace violence in Jordanian hospitals: A national survey. Int Emerg Nurs. 2016 Jan;24:61-5. doi: 10.1016/j.ienj.2015.06.005

19. Hsieh HF, Chen YM, Wang HH, Chang SC, Ma SC. Association among components of resilience and workplace violence-related depression among emergency department nurses in Taiwan: a cross-sectional study. J Clin Nurs. 2016 Sep;25(17-18):2639-47. doi: 10.1111/jocn. 1330920

20. de-San-Segundo M, Granizo JJ, Camacho I, Martínez-de-Aramayona MJ, Fernández M, Sánchez-Úriz MÁ. [A comparative study of aggression towards Primary Care and Hospital Health professionals in a Madrid health area (2009-2014)]. Semergen. 2017 Mar;43(2):8590. doi: 10.1016/j.semerg.2016.03.017.

21. Llor-Esteban B, Sánchez-Muñoz M, Ruiz-Hernández JA, Jiménez-Barbero JA. User violence towards nursing professionals in mental health services and emergency units. The European Journal of Psychology Applied to Legal Context 2017;9(1):33-40. http://dx. doi.org/10.1016/j.ejpal.2016.06.002.

22. Rafeea F, Al Ansari A, Abbas E, Elmusharaf K, Zeid M. Violence toward health workers in Bahrain Defense Force Royal Medical Services' emergency department. Open Access Emerg Med. 2017 Nov 8;9:113-121. doi: 10.2147/OAEM.S147982

23. Ogenler O, Yapici G. Assessment of physical violence against research assistants and nurses in an university hospital. Acta bioeth 2018 ; 24(1): 19-29. http://dx.doi.org/10.4067/S1726-569X2018000100019

24. Zahra A, Feng J. Workplace violence against nurses in Indonesian emergency departments. Enfermeria Clinica. 2018;28(Supl 1 Part A):184-190. doi: 10.1016/S1130-8621(18)30064-0.

25. Berlanda S, Pedrazza M, Fraizzoli M, de Cordova F. Addressing Risks of Violence against Healthcare Staff in Emergency Departments: The Effects of Job Satisfaction and Attachment Style. Biomed Res Int. 2019 May 28;2019:5430870. doi: 10.1155/2019 /5430870.

26. Shi L, Li G, Hao J, Wang W, Chen W, Liu S, et al. Psychological depletion in physicians and nurses exposed to workplace violence: a cross-sectional study using propensity score analysis. Int J Nurs Stud. 2020 Mar;103:103493. doi: 10.1016/j.ijnurstu.2019.103493.

27. National Institute for Occupational Safety and Health OHS. Nurses Are Suffering More Violence in the Workplace. Disponible en: https:/ / ohsonline.com/articles/2020/02/10/nurses-are-sufferingmore-violence-in-the-workplace.aspx. Acceso el día 10 febrero 2020.

28. Taylor J, Rew L. A systematic review of the literature workplace violence in the emergency department. J Clin Nurs. 2011 Apr;20(78):1072-85. doi: 10.1111/j.1365-2702.2010.03342.x.

29. Lanctôt N, Guay S. The aftermath of workplace violence among healthcare workers: A systematic literature review of the consequences. Aggress Violent Behav. 2014; 19:492-501. Doi: 10.1016/ j.avb.2014.07.010

30. Pourshaikhian M, Abolghasem H, Aryankhesal A, Khorasani-Zavareh D, Barati A. A Systematic Literature Review: Workplace Violence Against Emergency Medical Services Personnel. Arch Trauma Res 2016; 5(1):e28734. Doi: 10.5812/atr.28734. 
31. International Labour Office, International Council of Nurses, World Health Organisation, Public Services International. Joint programme on workplace violence in the health sector. Management of workplace violence victims. Jon Richards. Geneva 2003. P41.

32. Al-Maskari SA, Al-Busaidi IS, Al-Maskari MA. Workplace violence against emergency department nurses in Oman: a cross-sectional multi-institutional study. Int Nurs Rev. 2020 Jan 26. doi: 10.1111/ inr.12574

33. Hogarth K, Beattie J, Morphet J. Nurses' attitudes towards the reporting of violence in the emergency department. Australas Emerg Nurs J. 2016 May;19(2):75-81. doi: 10.1016/j. aenj.2015.03.006.
34. Wolf L, Delao A, Perthats C. Nothing changes, nobody cares: understanding the experience of emergency nurses physically or verbally assaulted while providing care. Journal of emergency nursing. 2013. doi:http://dx.doi.org/10.1016/J.jen.2013.11.006.

Manuscrito recibido el 7 de abril de 2020. Aceptado para su publicación, tras revisión, el 21 de julio de 2020.

\section{Workplace violence against emergency service nurses: an integrative review}

ABSTRACT

Keywords
Objective. Learn the worldwide frequency of physical and verbal violence against emergency service nurses, and its health and occupational impact.

Methods. An integrative review study was conducted in February 2020 of the MEDLINE, LILACS, CINAHL, and SciELO bibliographic databases, using the descriptors "Nurses," "Workplace Violence," "Physical Aggression," "Psychological Aggression," "Stress, Psychological," "Psychological Trauma," "Aggression," "Health Impact Assessment," "Health Impact," "Impacts on Health," "Impact Assessment, Health," and "Occupational Health." Results. Of the articles located, 18 were included in the analysis. The studies confirmed that the health workers most affected by violence were nurses, and the most common types of violence were verbal aggression, followed by physical violence. In the hospital, these assaults most often occurred in the emergency service. The correlated impact on nurses' health included stress, emotional exhaustion, anxiety, and fear, among other symptoms. With regard to occupational impact, significant indirect accounts related to the reduction in productivity were evident. All these aspects led nurses to consider leaving their jobs in emergency services.

Conclusions. The results enabled identifying a risk profile for violence against nurses in emergency services, seen in the high incidence of physical and verbal violence that led to reduced productivity on the job and the onset of symptoms of burnout syndrome.

Workplace violence; nurses; health impact assessment; occupational health; emergency medical services. 


\section{Violência no trabalho contra os profissionais de enfermagem em serviços de emergência: revisão integrativa}

RESUMO Objetivo. Conhecer a frequência mundial da violência física e verbal contra os profissionais de enfermagem nos serviços de emergência e seu impacto na saúde e no trabalho.

Métodos. A pesquisa foi realizada em fevereiro de 2020 por meio de uma revisão integrativa nas bases de dados MEDLINE, LILACS, CINAHL e SciELO utilizando os seguintes descritores: "Nurses", "Workplace Violence", "Physical Aggression", "Psychological Aggression", "Stress, Psychological", "Psychological Trauma", "Aggression", "Health Impact Assessment", "Health Impact", "Impacts on Health", "Impact Assessment, Health" e "Occupational Health".

Resultados. Dentre os artigos identificados, 18 foram incluídos na análise. Os estudos confirmaram que os profissionais da saúde mais afetados pela violência são os profissionais de enfermagem. Agressões verbais e violência física são as formas mais comuns de violência. O local dentro do hospital onde mais ocorrem as agressões é o serviço de emergência. O impacto na saúde dos profissionais de enfermagem está associado ao estresse, esgotamento emocional, ansiedade e medo, entre outros sintomas. Da perspectiva do trabalho, evidencia-se uma relação indireta significativa com queda de produtividade. Todos estes aspectos fazem com que os profissionais de enfermagem considerem desistir de trabalhar nos serviços de emergência.

Conclusões. Os resultados do estudo permitem identificar um perfil de risco de violência contra os profissionais de enfermagem nos serviços de emergência, evidenciado pela alta incidência de violência física e verbal que repercute com queda de produtividade no trabalho e surgimento de sintomas relacionados à síndrome de burnout.

Palavras-chave Violência no trabalho; enfermeiras e enfermeiros; avaliação do impacto na saúde; saúde do trabalhador; serviços médicos de emergência. 